\title{
A Study Investigating the Value of Dynamic Display and Browsing Experience of an e-Museum
}

\author{
Tingsheng Weng
}

\begin{abstract}
This study used the webpages of an e-museum to promote humanities and art works, and tested the subjects' assessment of humanities literacy, webpage design, and visual perception. The analysis methods include the collection of relevant studies and the use of a questionnaire survey to arrange and summarize the data. The research results showed that most of the subjects, who are of the digital generation, spoke highly of humanities literacy. The subjects strongly agreed that relevant links provided by the webpages can help them complete their art knowledge. In terms of visual perception, they indicated that screen layout, color matching, and dynamic interpretation can effectively attract the attention of users when viewing webpages, thus, enhancing their absorption of art knowledge.
\end{abstract}

Index Terms-Dynamic webpages, dynamic e-books, visual perception, humanities literacy, webpage design, digital generation.

\section{INTRODUCTION}

In addition to attaching importance to learning professional knowledge and skill training, college education should integrate information technology and humanities literacy into education to expand students' spiritual world and cultivate their sound personality. Education should give priority to effectively improving teaching quality, reconstructing social order, and restructuring human value and humanities literacy to further expand the outlook on life in this new e-century [1]. The first issue discussed at the $8^{\text {th }}$ National Science and Technology Conference, as convened by Taiwan's Ministry of Education [2], was to integrate humanities technology and to improve the education system for life quality. The conference noted that students majoring in humanities sciences lack information technology and technological knowledge, while those majoring in science and technology lack humanities literacy. This phenomenon leads to a failure to completely integrate knowledge and a lack of cultural insights in the development of relevant competitive information technology-related industries in Taiwan. Therefore, the competitive advantage of the nation's technical capacity cannot be further enhanced.

The technological literacy of students majoring in humanities sciences is generally low, and they fail to use technology to benefit their learning in the field of humanities, research, and innovation, while students in the field of technology fail to meet social needs due to their lack of

Manuscript received Apr. 12, 2017; revised October 25, 2017.

Tingsheng Weng is with the Department of Management Business Administration, National Chiayi University, Taiwan (e-mail: politeweng@mail.ncyu.edu.tw). humanities literacy. The only way to improve this situation is to develop comprehensive technological literacy and humanities literacy of the people in Taiwan. Only when humanities content is integrated with technology can e-learning products with high value-added benefits be developed. The E-learning of end-users is found to be beneficial for the development process of teaching and learning materials, in order to increase the usability and quality of developed materials, which also satisfies such end-users [3].

The important measures promoted by Taiwan's Ministry of Education [2] include: 1) use technology to establish environments for a digital audience; 2) cultivate talents with both technological and humanities literacy; 3) develop digital teaching materials that integrate humanities with technologies. Therefore, it is important to integrate information technology with humanities literacy in Taiwan's education system.

Take the U.S. for example, while it has been a nation for just slightly over 230 years, its abundant humanities literacy and technological development are worthy of learning and following by all countries around the world. There are many museums and memorials around its capital, Washington, D.C., and it would take several weeks or more to experience each them attentively. Therefore, visitors' desire for knowledge cannot be satisfied by only several days in the capital. If visitors intend to fully understand the museums, then entrance fees will also be a heavy burden.

Museums are not only for the sake of preserving cultural heritages, but can also make information content accessible to the wider public in an attractive manner. Emerging technologies are widely used to create virtual museum exhibitions, both in a museum environment through informative kiosks and on the World Wide Web [4]. In terms of the development trend of museums, interactive exhibitions and educational activities are now generally provided. While the aim of museums is to provide national education, the exhibition halls of traditional museums cannot be designed like other types of exhibitions. However, visitors can freely browse and easily interact with e-museums, which can be designed to provide even more diversified services to meet the needs of the public than traditional museums can satisfy.

Museums in Europe are attempting to address some of these problems by developing museum templates for open-source content management systems (CMSs), such as Joomla 1.5. Such templates, when downloaded online, allow small- and medium-size institutions to create high quality portals for content dissemination on the Internet with low economic investment and computer expertise. Curators can easily manage and update contents, including new 
functionalities, and personalized and scale portals, which favor the introduction of Web 2.0 applications. Group Òliba is currently developing such open-source templates for Catalan museums and cultural associations, which will be available online free of charge for any institution [5].

With the advances of digital technology and general applications of the Internet, the emergence of information technology has led to significant changes in the transmission of humanities and knowledge. "Digitalization" has become the main strategy comprehensively adopted by various major museums around the world. Fang, Wang, Chang, and Fan [6] noted that the development of digital technology has had a profound influence on the design of museum exhibitions; however, the application of mobile technology is still open for further development regarding the audience learning experience in museums. For example, the British Museum, the Louvre, Memory of the World of the United Nations, American Memory of the U.S., the National museums [7] of Canada, and the National Palace Museum in Taiwan have all established e-museums for economic, educational, and research functions, as well as to promote abundant cultural assets. In fact, the last one created the "National Palace Museum Cultural Industry Park" as its platform for the revitalization of Taiwan's cultural and creative industries [8]. If collected humanities and arts works can be integrated with e-technology, new forms of innovation can be expanded and diversified education can be developed to achieve cross-space and time, as well as cross-border and cross-sectoral applications. The developmental benefits of humanities value can be improved if the content and knowledge of humanities and arts are integrated with technology through the use of e-technology.

The current trends of teaching and learning with web education lead to better learning environments [9], [10]. Progress in information technology also has led to further advances in the ways of reading. The integration of webpages with e-books has resulted in a revolution of e-reading, thus, forming its future expansion trend.

\section{RESEARCH MOTIVES}

In this era of information technology and multimedia, museums are a good place for developing and utilizing learning resources, thus, helping to develop an information society and offering equal opportunities for everybody [11]. Museums also follow the trend of the digital era in utilizing the transmission of e-learning multimedia to attract a greater learning population. Although there are no entities collected in e-museums, e-museums convert entities into digital objects as digital archives. Digital exhibitions and educational objectives can expand the benefits of diversified value-added applications and shorten the distance between the public and exhibits, hence, achieving the ideal objective of museums. E-museums are an engaging medium with great appeal to a variety of groups of people, and can promote the 'real site' by providing information about museum exhibitions and offering enhanced displays of museum artifacts through emerging technologies [12].

To understand e-museum consumers' use and cognitive behavior in the utilization of digital media technologies, this study employs digitalization to build a webpage platform of an e-museum. General websites display artworks via the layout of fixed texts and images; however, if the contents contain too many texts, then users have to move a mouse to drag the layout or click on the next page to browse the complete texts of the content. Therefore, the webpage content herein is displayed via marquee autoplay to enable users to browse the complete content of the same page via a marquee autoplay. The research motives of this study are to investigate the content displayed via this marquee autoplay, users' acceptance of the smoothness of browsing, the comfort of color rendering, and how an e-museum attracts the public's interest in browsing.

\section{RESEARCH PURPOSES}

With increased awareness of cultural preservation and the gradual maturation of digitalization technologies, an increasing number of e-museums have been established, and such e-museums with large collections are particularly devoted to e-archiving, and triggering the domestic and global trend of e-archiving [13]. As such, the e-museum set up in this study can archive arts and cultural works, which be utilized to examine users' dissatisfaction under the three dimensions of humanities literacy, webpage design, and visual perception, as well as to assess the effectiveness of browsing e-museums. This study conducts a questionnaire survey, performs analyses, and determines users' level of recognition and care for the perceived e-museum content. This study also offers conclusions and suggestions as reference for the future adjustments and improvement directions of e-museums, humanities, and arts platforms.

\section{LITERATURE REVIEW}

\section{A. Humanities Literacy}

Kuo [14] suggested that humanities literacy should include two aspects: knowledge and attitude. Students of various departments and institutes should receive humanities education aimed at cultivating humanities literacy and developing humanities spirit. The insights into humanities literacy should include: 1) pondering the various critical issues of mankind in an in-depth, clear, comprehensive, and logical manner; 2) understanding how to appreciate and evaluate classical, arts, and literary works, and to admire the spiritual heritage of mankind and use it to enrich one's personal life; 3 ) realizing the rules of getting on in the world and to maintain an open mind.

Chen and Lin [15] suggested that "humanities literacy is a value of life, an attitude of life, and a life guide used to enable individuals to recognize their direction in life, know how to live, how to accept or reject, and how to bring out the value of life. Individuals with humanities literacy possess an open-minded attitude, broad vision, careful thinking, and integral understanding, and are able to make reasonable decisions and choices to become those who are knowledgeable, sensible, can distinguish right from wrong, and understand the interest or well-being of the larger group". 
Lin and Chen [16] noted that humanities literacy includes three dimensions: knowledge, attitude, and ability. The most important goal of humanities literacy is to enable students to possess an abundant amount of humanities and social knowledge, as well as to understand their own social development. Once they are nurtured by humanities education, and develop an accurate value of life and an attitude of care for mankind and society, their abilities of interpersonal communication, critical thinking, artistic appreciation, cultural comparison, adaptation to change, and self-reflection can be cultivated.

Drigas, Koukianakis, and Glentzes [17] stated that the digitization of arts and culture refers to the relationship between ICTs and the production and consumption of art and culture. Zong and Wang [18] presented that visual literacy as humanities literacy is not simply employed for technology indoctrination and skills to operate, as the web interface, as a tool of visual information, has an important role in the dissemination of visual literacy, and can help cultivate visual literacy to provide a new visual platform. This study targets the recognition of e-museum users' level of social concern, cultural understanding, and the meaning of life, as reflected by their thinking and actions [19]. In the current information knowledge economy, the importance of humanities to students is no less than that of professional knowledge and technology. One of the dimensions investigated herein is how young people perceive humanities literacy when using an e-museum website in the category of digital humanities and arts.

In the past, different research studies found that the term "Internet literacy" mostly referred to a network, as well as the network's ability to use knowledge. However, with the rapid progress of network technology, its modern meaning is constantly changing, as text is being added to the concept, etiquette, and ethics, including whether the user knows how to have discipline on the network, not infringe on the rights of others, and avoid violating the law of norms [20].

While a wide spread of behaviors can benefit many people, wide spread behavior that lacks a "humanity" network, such as manufacturing a network virus, spreading hate, false, or malicious attacks, or denigrating freedom of speech, will be spread throughout history. "Network humanities" include Internet ethics, netiquette, legal knowledge, and all networks that are required to follow the basic principles of behavior, and by "respecting" the foundation that carries everyone's network behavior [20].

What we are trying to state is that, things on a network platform must be published after review, in order to transmit the benefits to more people, share information across various peoples, and generate outcomes from sharing such information. Thus, the present study is based on strong humanities literacy, and takes a a web version of a painting in a museum, like that of a spindle. The positive benefits of this study are to promote communities and enhance public participation in humanities literacy.

\section{B. Webpage Design}

Following the advances in information technology and the development of the Internet, the performance of webpage design has been increasingly diversified, as such performance and the use of design elements have varied with technological evolution and media reform. Veen [21] divided webpage design into library-based design and gallery-based design. The former is a function-based design mainly composed of texts that mostly provides information; therefore, it attaches less importance to the style and aesthetic feeling presented by information, such as Google and Yahoo search engines. On the contrary, the latter design of image interfaces or metaphors is a form-based design, which attaches greater importance to color, creativity, and atmosphere building [22].

With the popularity of Internet information, employing information technology to establish e-museums [23] has become the developmental trend of traditional museums. Chia [23] suggested that e-museums encompass a newly-arising field of knowledge interpretation and management, an online knowledge treasury, a new challenge for the e-generation to bring Internet resources to teaching, and an ability required to be learned. Therefore, this study designs an e-museum, which it provides to students attending day schools, night schools, and holiday classes for browsing, in order to conduct our investigation.

Chen, Li, and Liao [24] mentioned that webpage design must provide users with complete information, such as easy-to-use information, useful information, and entertainment, as well as delivering vivid animation effects and offering relevant image content to provoke further interest. Webpage design refers to the close integration among the elements used in the layout design, such as colors, images, navigation system, and information arrangement [25]. By using the rich medium of web pages as our input, aesthetic appeal is captured by a metric that considers the placement of all objects on the screen. The placement of images is a strong predictor of both aesthetic appeal and perceived usability [26]. Webpages should also include dynamic and static media effects that enable users to notice the individual images or functions of websites, as well as to better perceive the visual experiences of the overall atmosphere. To understand how users perceive the elements used in webpage design, such as colors, images, dynamic media, navigation system, and information arrangement, this study enables them to notice the individual images or functions of the website, and to peruse the overall atmosphere provided by the website, which is Dimension 2, to be examined in this study.

\section{Visual Perception}

People react to the visual cues of colors, forms, and movements [27]. Shih [28] suggested that visual perception refers to specific light waves reflected or transmitted through objects, which become the source of visual stimuli. These messages are transmitted to the brain for analysis and interpretation to affect a person's awareness and behavioral performance.

Webpage visual perception also plays the role of information and user communication. Jedi [29] mentioned that, with the increasingly diversified content and elements of webpages, designers should ensure that users can receive accurate messages transmitted from the website, and grasp the ideas, abilities, and responses of users, which will help in 
creating webpages and enable users to perceive more abundant values through multimedia webpage browsing.

Peoples' perceptions of a website mainly originate from whether they can achieve the objective of visiting it. User experience refers to the sum of feelings and thoughts when browsing a website. If users' experience is positive, they should obtain a sense of accomplishment and satisfaction. If it is negative, the residual feelings of frustration and anger will prevent them from revisiting that website. Therefore, designers of information websites should consider and understand the user experience of the digital generation, present the necessary texts and images, and employ adequate visual designs for specific functionality to achieve high effectiveness. During the building and design of a website, it is necessary to anticipate the potential needs and attitudes regarding the use of screen layout, color matching, and dynamic interpretation to visitors of the digital generation who may browse the website.

To understand users' perceived effectiveness of the multimedia effect in their visual perception via an e-museum, this study transmits introductory messages regarding the insight and knowledge of the artworks to users for their own analysis and interpretation, in order to see how dynamic interpretation, layout, and color matching affect the awareness and satisfaction of the new digital generation, which is Dimension 3 to be investigated in this study.

Many past studies have researched the quality of webpage design, with most considering easy usability as a priority factor (Davis et al. [30]; Chen \& William [31]; Brackett \& Carr [32]; Lynn et al. [33]; Schlosser et al. [34]). The second factor that should be considered is the usefulness of the information (Davis et al. [30]; Cary [35]; Wan et al. [36]; Brackett \& Carr [32]; Lynn et al. [33]; Schlosser et al. [34]). For example, Wan et al. [36] assessed research subjects' website construction, considered information enrichment, public information, and online transactions, and provided a variety of methods of information indexing, query functions, and guiding functions, which all offer positive benefits to marketing website pages [37].

\section{RESEARCH METHOD}

Literature review shows the importance of humanities literacy, webpage design, and visual perception. Therefore, this research first uses E-learning multimedia applications in an e-museum to allow readers to read it easily, and then constructs a website system to include more readers. After readers browse the site by E-learning multimedia applications and the website system, this study designs a related questionnaire, including the types of humanities literacy, webpage design, visual perception, and semi-structured questions regarding three dimensions. Finally, this study conducts research analysis, and Fig. 1 presents the research process.

\section{A. Website Design}

A layout with texts and images concurrently presented is adopted in the design of the e-museum website, and presented on the website in an e-book manner. The layout is divided into 2 pages. The left page has the image of a fixed artwork, while the right page has the description content (Fig. 2). The Q\&A content for testing users' level of understanding the images after browsing is presented via a marquee autoplay. The text is played from the bottom to top, and all the descriptive contents are autoplayed. When users turn to the next page to browse the next piece of artwork, the presentation is the same.
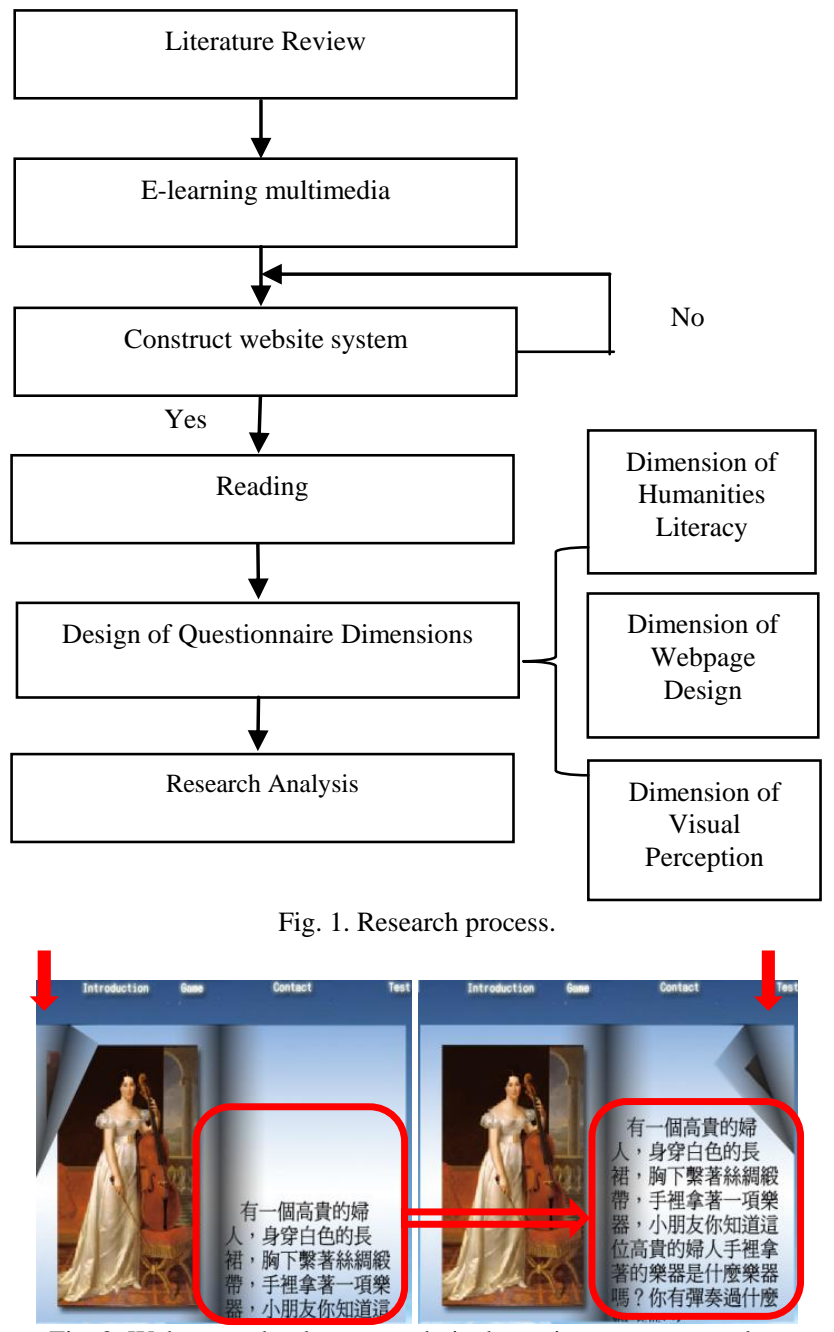

Fig. 2. Webpage e-book presented via dynamic marquee autoplay.

\section{B. Research Subjects}

The research subjects are the students of a digital technology department who are accustomed to Internet operations.

In addition to cultivating a professional labor force and giving consideration to both theories and practices, college education should attach importance to personality cultivation, cultural nurturing, and the development of students' accurate occupational ethics. Lin and Chen [16] reviewed the courses of junior colleges, and found that they place particular stress on professional skills, while ignoring cultural nurturing. Therefore, contemporary students lack the knowledge of life etiquette and a sense of responsibility, and exhibit the problematic behaviors of moral deviation.

This study integrates humanities with professional knowledge in the courses of information management and implements teaching activities, in order to effectively train students of the digital generation to use information media websites. The study also targets improving literacy of the 
humanities, in order to increase students' concern for mankind and society, which are important issues.

\section{Design of Questionnaire Dimensions}

This study performs analyses and investigations according to literature review, and sets up items in 3 dimensions: humanities literacy, webpage design, and visual perception.

H1 Dimension of Humanities Literacy

H1.1 I can accept various kinds of art and culture.

H1.2 I feel that sample works with both images and texts can help me learn.

H1.3 I am able to analyze works.

H1.4 I frequently participate in various artistic and cultural activities.

H1.5 I feel that browsing the artistic and cultural content of an e-museum can improve an individual's humanities literacy.

H1.6 I feel that browsing an e-museum enables me to increase my individual concern for society and reflect it in my life and behavior.

As schools are units of talent cultivation, and both the government and the Ministry of Education are promoting humanities literacy, the following two items are added.

$\mathrm{H} 1.7 \mathrm{I}$ feel that talent cultivation is an important objective of school education.

H1.8 I feel that the government has shown respect to the pluralistic development of art and culture.

H2 Dimension of Webpage Design

H2.1 The design of the operating instructions of the e-museum are comprehensible.

$\mathrm{H} 2.2$ It is easy to operate the website interface.

H2.3 There are audio introductions that enable people to obtain clear information.

H2.4 The webpage content helps people to better understand the artistic and cultural background of paintings in this century.

H2.5 The webpage links of relevant works enable people to better understand the works.

H3 Dimension of Visual Perception

H3.1 The dynamic presentation of text from bottom to top via the marquee gives a detailed explanation about the works' content.

H3.2 The webpage layout can be easily identified and smoothly used.

H3.3 The presentation of colors makes people feel comfortable.

H3.4 The overall layout is attractive.

\section{RESEARCH ANALYSIS}

During this study, the e-museum website is provided to 101 students for browsing, and the students could complete the questionnaires online. A total of 80 questionnaires are returned, with 79 valid samples. The analyses are summarized, as follows.

\section{A. Analysis of the Background Information of Samples}

According to the results, as summarized from the valid samples, analysis shows that: 1) $70 \%$ of the subjects are female; 2 ) most of the subjects are from central and southern
Taiwan (89\%); 3) $98 \%$ of the subjects are under the age of 30; 4) $96 \%$ of the subjects are students. In general, young students have more leisure time versus other age groups and workers. However, on average, the subjects only visited real museums in person 1-2 times every year.

The data shows that cultivating humanities literacy in the people of Taiwan still requires many more efforts and improvements. Therefore, this study suggests that, during the promotion of improving humanities literacy, the government and education authority should consider that the cost of visiting real museums in person may be a burden. Consequently, the knowledge literacy of humanities collections can be added to those places where e-museums are promoted.

\section{B. Analysis on Humanities Literacy}

The data of the survey respondents shows that they are a digital generation, as the acceptance rate of $81 \%$ for the various kinds of artistic and cultural knowledge by the subjects is quite high. In fact, $61 \%$ suggest that the government has shown respect to the pluralistic development of art and culture; $80 \%$ suggest that participating in artistic and cultural activities can increase individuals' humanities literacy and raise their concern for society, as reflected in their life and behaviors; $68 \%$ suggest that the example works of texts and images can help them learn; $34 \%$ are able to analyze artworks; however, only $29 \%$ have participated in relevant artistic and cultural activities.

While the subjects' usual participation in artistic and cultural activities, as well as their ability to analyze artworks, is not high, they have affirmative attitudes towards accepting different art and cultures, suggesting that many of them usually lack the opportunity and habit to participate in artistic and cultural activities. Increasing attention has been attached to humanities literacy, suggesting that the young members of the digital generation can appreciate art, compare cultures, engage in critical thinking, and show concern for humankind and society, and have been cultivated within Taiwan's social environment.

Most of the subjects' assessment scores for "The government has shown respect to the development of art" are neutral. Therefore, the government should make efforts to improve this issue. Only when the government provides more space for artistic creation can more people engage in its promotion for the routine purpose of cultivating national humanities literacy.

\section{Analysis on Webpage Design}

From analysis of the collected data, more than $55 \%$ of the subjects positively commented on the relevant webpage links, audio introduction function, and the use of a game to test their perception of art.

[The layout is attractive, which is a very good thing.]

[The overall design is good.]

[The webpage is created attentively.]

[Vivid and lively.] 
Sixty percent agree that art-related cultural messages can be understood through this e-museum.

[Enables users to notice the insights of the individual artistic images of the website.]

Sixty percent agreed that audio forums are important. Some subjects suggested the following.

[I think that blind people need more audio design.]

Sixty-eight percent agreed with the addition of links to relevant works.

Regarding relevant webpage links, the audio introduction function, and the use of a game to test the perceptions of art, the webpages of the e-museum exhibit vivid and dynamic effects, provide relevant image content, and enable most users to easily compare the insights and meanings of humanities and arts in the images and texts while browsing through the guided questions and the comparison design [image] [text]. They feel that the site aroused their interest and helped them to understand and perceive the overall atmosphere provided by the website, as well as the links to relevant works.

Lin [7] previously served as the Researcher and Director of the Exhibition Service Department of the National Palace Museum, and said, "The museum has faced many operating challenges with the continuous innovation and social changes of digital technology. If a museum can grasp the key future trends and challenges, use technology to interpret abundant cultural knowledge systems, and form interesting learning resources and an intellectual interactive environment, it can create a new vision for the application of museum technologies."

The research results can be provided as reference for relevant museums to improve their own e-webpages, and design them more dynamically to meet the browsing needs of the digital generation, thus, increasing their usage intention, and laying an important foundation for the promotion of art and culture.

\section{Analysis on Visual Perception}

About $60 \%$ of the subjects have positive comments regarding the screen layout, color matching, and dynamic interpretation of the e-museum. The subjects suggest that the function of dynamic interpretation is complete and the speed of interpretation is adequate, which helps them understand the works. Some of the subjects suggest the following.

[People are most susceptible and sensitive to visual perception. How to use colors and animations to attract the eyes of people is the most important.]

[Visual perception refers to certain specific light waves reflected or transmitted through objects, which become the source of visual stimuli. These messages are transmitted to the brain for analysis and interpretation to affect users' awareness and behavioral performance.]

In other words, the screen layout, color matching, and dynamic presentation of an e-museum could attract subjects' attention and guide them to browse the various contents. No residual feelings of frustration, anger, or negative user experiences remained. According to the answers to the open-ended questions, most subjects are satisfied with the visual perceptions of the e-museum, and suggest that the colors, animation design, etc. could effectively attract users' attention [to browsing].

Based on the above, visual perception is able to attract people's attention during browsing to improve their absorption of artistic knowledge, or put differently, there is web accessibility in an e-museum. Webpage designers should be aware of accessibility-related issues, and properly express the contents and meanings of the webpages, in order to improve user experience. For example, they should design practical presentation methods for different image contents according to the meanings of the artistic and cultural works. In addition, it is necessary to consider the visual perceptions of the various user roles. The results of the questionnaire survey can guide webpage designers to review their webpages, provide proper accessibility, and understand the actual effects of the contents.

\section{CONCLUSION AND SUGGESTIONS}

In order to improve national competitiveness, establish an island encompassing humanities technology, and develop national technologies, it is necessary to raise citizens' "humanities literacy" and improve their quality of life.

According to the feedback from this study, most people of the digital generation had positive comments about humanities and artistic literacy, and suggested that individual concern for society and an understanding of culture are important indicators of the progress of humans and social civilization. Some subjects suggested that schools, the government, and society should promote more humanities activities and cultivate artistic talents through e-technology. Moreover, it is advised to update the regional artistic and cultural layout in order to improve regional e-development.

Utilizing the dynamic features of a marquee in the building of webpages can help distinguish the presentation of other webpages and increase the need for browsing by other types of users. The digital generation is accustomed to browsing e-webpages on a daily or even hourly basis. The instruction of the design skills of e-webpages can be integrated into school education, in order to strengthen the cultivation of future talents in artistic technologies.

Only when the government provides more space for artistic creation can more people engage in art promotion for the routine purpose of cultivating national humanities literacy. Regional governments can develop artistic and cultural facilities with regional characteristics as a form of marketing through artistic and cultural e-museums Governments and society can thus use e-webpages more often to promote artistic and cultural activities.

Museums are places for the development, provision, and utilization of learning resources. In order to respond to the social changes of the information technology era and the diversification of learning patterns, as well as to serve more users of e-museums, it is advised to separately add functions 
of necessary special effects design, art design, and multinational/lingual audio guides of unit works to webpage contents according to the necessary scope. This will help expand the comprehensible utilization of e-museums and enrich the multi-ethnic culture of e-marketing. Information technology can help develop arts and humanities education to become a part of social education, bringing the country one step closer towards the ultimate goals of holistic education and humanities literacy.

\section{ACKNOWLEDGEMENT}

The author would like to thank Chi Mei Museum for their help in this research project.

\section{REFERENCES}

[1] Y. H. Chu, "A study on the cultivation of technological literacy from the perspective of holistic education of e-generation," Living Technology Education, vol. 35, no. 12, 2002, pp.2-12.

[2] The first issue discussed in the 8th National Science and Technology Conference convened in Taiwan Ministry of Education: To integrate humanities technology and to humanities technology and to improve the education system of life quality, Sub-issue 5: To improve national literacy and infrastructure of public knowledge, 2009, Sub-issue $1 \mathrm{pp}$. 5-1-5.

[3] R. Rahamat, P. M. Shah, R. Din, S. N. Puteh, J. A. Aziz, H. Norman, and M. A. Embi, "Measuring leaners' perceived satisfaction towards e-learning materials and environment," WSEAS Transactions on Advances in Engineering Education, vol. 9, no. 3, 2012, pp. 73-83.

[4] S. Sylaiou et al., "Virtual museums, a survey and some issues for consideration," Journal of Cultural Heritage, vol. 10, no. 4, 2009, pp. $520-528$.

[5] P. Báscones and C. Carreras, "Project Mus. cat: A CMS open-source application," International Journal of Web Based Communities, vol. 8, issue $1,2012$.

[6] Y. F. Rong et al., "Develop a mobile learning model for museums," presented at the 6th WSEAS International Conference on Applied Computer Science, Hangzhou, China, April 15-17, 2007.

[7] Q. P. Lin, "Adoption horizons: Museum technology foresight," Museology Quarterly, vol. 23, no. 3, 2009, pp. 5-15.

[8] T. S. Han, "A study of the new internet platform of the national palace museum and its effects on users satisfaction and brand equity," NSC101-2420-H004-007-SS3, 2012.

[9] R. Rahamat, P. M. Shah, and R. Din, "Measuring learners' perceived satisfaction towards e-learning material and environment," WSEAS Transactions on Advances in Engineering Education, issue 3, vol. 9, July, 2012, pp. 72-83.

[10] J. A. Aziz, R. Din, P. M. Shah, R. Rahamat, S. Z. A. Manaf, and M. A. Embi, "Collaborative filtering for teaching in a learning 3.0 environment," in Proc. the 10th WSEAS e-Activities, 2011.

[11] P.-R. Brandusa, "Innovative pedagogical intervention strategies and social software technologies in an e-learning project initiated by the University Politehnica of Bucharest," (Faculty of Applied Sciences), WSEAS Transactions on Advances in Engineering Education, issue 1, vol. 7, January, 2010, pp. 11-20.

[12] P. Patias et al., "The development of an e-museum for contemporary arts," Conference on Virtual Systems and Multimedia, 2008.

[13] M. D. Wu and K. L. Hsu, "An analysis of educational websites of digital libraries and digital museums," Library Association of Republic of China (Taiwan) Conference Report, no. 75, 2005, pp. 37-62.

[14] W. F. Kuo, Humanities Education in Technology Era (3rd ed.), Taipei: Youth Culture, 1993, pp. 189-191.

[15] Y. H. Chen and H. L. Lin, "To strengthen the concept of humanities literacy education in technical and vocational schools," in Proc. $16^{\text {th }}$ National Conference on Technical and Vocational Education: Category of General Technical, Vocational and Humanities Education: Group of Humanities Education, 2001, pp. 306-312.

[16] Y. L. Lin and H. Y. Chen, "Improving the humanity integrity of students of technological college and university," Journal of China Institute of Technology, vol. 30, 2004, pp. 67-76.
[17] D. Athanasios et al., "An e-culture-e-museums environment for common citizens and disabled individuals," Int. J. of Digital Culture and Electronic Tourism, vol. 1, no. 4, 2009

[18] Z. S. Ying and W. Ning, "Develop visual literacy web interface visual principles," Modern Distance Education, issue 5, 2010, pp. 68-71.

[19] Y. T. Lung, Refection of 100 Years, Taipei: China Times Publishing Company, 1999, pp. 18-19.

[20] Y.-P. Lin and C.-F. Tseng, "Cruise in the cyberspace - The necessity of internet humanity to avoid improper information exposure," Tzu Chi Nursing Journal, vol. 10, no. 1, 2011.

[21] J. Veen, Hot Wired Style: Principle for Building Smart Web Sites, Wired Book Inc, San Francisco, California, 1997.

[22] C. C. Chang and H. C. Yang, "Market positioning of websites - The case of music website homepage," Journal of Design, vol. 11, no. 3, 2006, pp. 81-97.

[23] T. M. Chia, "Digital museum application and learning for elementary and secondary school teachers," Museology Quarterly, vol. 19, no. 2, 2005, pp. 107-117.

[24] L. C. Chen et al., "The effects of science museum website design quality, attitude certainty on consumers' purchasing intention," Technology Museum Review, vol. 14, no. 1, 2010, pp. 3-23.

[25] C. C. Lin et al., "The study of consumer cognition on the commercial product information display of webpage," Journal of Applied Art and Design, no. 6, 2012, pp. 1-16.

[26] H. C. Purchase et al., "Investigating objective measures of web page aesthetics and usability," in Proc. the Twelfth Australasian User Interface Conference, 2011, pp. 19-28.

[27] P. M. Lester, Visual Communication Image with Messages 6e, USA Wadsworth publishing company, 2011, pp. 1-448.

[28] S.-W. Shih, Principles of Interior Lighting Design, Taipei: Shu Hsin Publishing, 1996.

[29] Jedi, Building Webpages with Accessibility: Building of Multimedia Webpages Accessible to Everyone, Taipei: Sung Gang Asset Management Corp. Limited, 2012, pp. 1-488.

[30] F. D. Davis et al., "User acceptance of computer technology: A comparison of two theoretical models," Management Science, vol. 35, no. 8,1989 , pp. $982-1003$.

[31] Q. Chen and D. W. William, "Attitude toward the site," Journal of Advertising Research, vol. 39 no. 5, 1999, pp. 27-37.

[32] L. K. Brackett and B. N. Carr, "Cyberspace advertising VS. other media: Consumer VS. mature student attitudes," Journal of Advertising Research, vol. 41, no. 5, 2001, pp. 23-32.

[33] G. S. Lynn et al., "Factors impacting the adoption and effectiveness of the world wide web in marketing," Industrial Marketing Management, vol. 31, 2003, pp. 35-49.

[34] A. E. Schlosser et al., "Converting web site visitors into buyers: How web site investment increases consumer trusting beliefs and online purchase intentions," Journal of Marketing, vol. 70, April, 2006, pp. 133-148.

[35] C. C. Cary, "Designing effective bed \& breakfast web sites," Proceeding of Fifth Annual Graduate Education and Graduate Students, Research Conference in Hospitality \& Tourism, Houston, USA, 1999, pp. 430-432.

[36] C.-S. Wan et al., "A study of web site content analysis of international tourist hotels and tour wholesalers in Taiwan," in Proc. the Fifth Annual Graduate Education Students, Research Conference in Hospitality \& Tourism, Houston, USA, 2000, pp. 132-137.

[37] L. C. Chen et al., "The effects of science museum website design quality, attitude certainty on consumers purchasing intention," Technology Museum Review, March, 2010, vol. 14, no. 1.

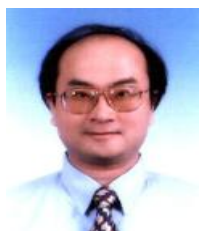

Ting Sheng Weng is an associate professor of the Department of Business Administration at National Chiayi University, Taiwan. He received his Ph. D. in division of management and information science, Graduate School of Advanced Technology from Kinki University, Japan in 2000. He had served as visiting professor at Meiji University in Japan from 2006 through 2007. His major research interests include science technology and management, e-learning, information education, technology education, MIS, and image processing 